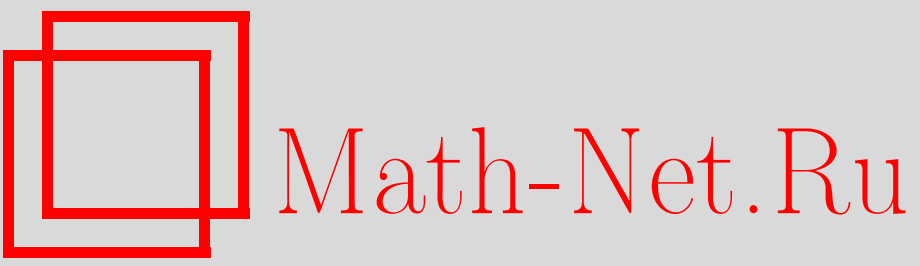

В. Р. Халилов, Квантовые макроскопические эффекты в вырожденном сильно намагниченном газе нуклонов, ТМФ, 2002, том 133, номер 1, 103-120

DOI: https://doi.org/10.4213/tmf383

Использование Общероссийского математического портала Math-Net.Ru подразумевает, что вы прочитали и согласны с пользовательским соглашением

http://www.mathnet.ru/rus/agreement

Параметры загрузки:

IP : 54.224 .60 .19

26 апреля 2023 г., 11:25:25 
ТЕОРЕТИЧЕСКАЯ

И МАТЕМАТИЧЕСКАЯ

ФИЗИКА

Том 133, № 1

октябрь, 2002

(C) 2002 г.

В.Р. Халилов*

\title{
КВАНТОВЫЕ МАКРОСКОПИЧЕСКИЕ ЭФФЕКТЫ В ВЫРОЖДЕННОМ СИЛЬНО НАМАГНИЧЕННОМ ГАЗЕ НУКЛОНОВ
}

\begin{abstract}
Модель вырожденного газа нейтронов, находящихся в химическом равновесии с вырожденными протонами и электронами в постоянном однородном сверхсильном магнитном поле, применяется для описания состояния вещества в центральных областях сильно намагниченных нейтронных звезд. Получены выражения для термодинамических величин (плотностей энергии и числа частиц, давления, намагниченности) вырожденного газа нейтронов, протонов и электронов с учетом вкладов, обусловленных взаимодействием аномальных магнитных моментов фермионов с магнитным полем. Обсуждаются макроскопические эффекты, которые могут иметь место в сильно намагниченных нейтронных звездах. Показано, что в сильном магнитном поле все термодинамические величины, характеризующие электрически заряженные фермионы, должны испытывать непериодические осцилляции, которые обусловлены взаимодействием аномальных магнитных моментов протонов и электронов с магнитным полем. Также показано, что уже при относительно невысоких плотностях нуклонов и электронов, зависящих от величины магнитного поля, все фермионы становятся полностью поляризованными по спину. Эффект полной спиновой поляризации нейтронов обусловлен взаимодействием аномального магнитного момента нейтрона с магнитным полем. Полученные результаты могут оказаться полезными для понимания процессов, протекающих в ядре нейтронной звезды с "вмороженным" магнитным полем.
\end{abstract}

Ключевые слова: энергия Ферми, аномальный магнитный момент, термодинамические величины, полная спиновая поляризация, магнитные осцилляции.

\section{1. ВВЕДЕНИЕ}

В последние годы внимание физиков-теоретиков и астрофизиков привлекают модели макроскопических тел, состояших в основном из нейтронов в сверхсильном магнитном поле $B$. Намагниченную нейтронную звезду, обладаюшую экстремально сильным магнитным полем, называют магнитаром (magnetars) [1], [2]. Как предполагают [3], [4], напряженность магнитного поля на поверхности экстремально намагниченной нейтронной звезды может быть порядка $B \geqslant 10^{15}$ Гс. Условие стабильности нейтронной звезды

* Московский государственный университет, Москва, Россия. E-mail: khalilov@thc.phys.msu.su 
налагает ограничение на максимально допустимое значение внутреннего магнитного поля, “вмороженного" в звезду, вытекаюшее из скалярной теоремы вириала [5]. При этом, если магнитное поле $B$ на поверхности нейтронной звезды имеет порядок $10^{15} \Gamma$, то в центральной области звезды магнитная индукция может стать порядка $10^{18} \Gamma \mathrm{c}[6]$.

Сушествование "вмороженного" в звезду сверхсильного магнитного поля приводит к увеличению значения максимально возможной массы стабильной нейтронной звезды по сравнению с ее величиной без магнитного поля [7]. Магнитное поле должно оказывать существенное влияние на микропроцессы с участием элементарных частищ, которые могут протекать в нейтронной звезде, и особенно на процессы в ее центральной области [8]-[13]. В работах [14], [15] изучалось влияние сильного магнитного поля на некоторые свойства вырожденного плотного газа нуклонов, в котором мезонное взаимодействие между нуклонами учитывалось в так называемом “приближении среднего поля", т.е. в приближении внешнего постоянного и однородного (мезонного) поля.

В работах [16]-[18] численными методами изучались уравнение состояния и намагниченность вырожденного идеального газа электронов и нуклонов в присутствии сильного постоянного магнитного поля с учетом аномальных магнитных моментов (AMМ) электронов и нуклонов. Было показано, что эффекты квантования энергии и орбит поперечного (вектору напряженности поля) движения заряженных частиц в постоянном и однородном магнитном поле играют заметную роль и, в частности, приводят к увеличению равновесной плотности протонов по сравнению со свободным случаем [16], [17]. Однако в работах [16], [17] не обсуждались эффекты, обусловленные, например, анизотропией полного тензора напряжений вещества и магнитного поля, а в [18] эти эффекты исследованы некорректно.

В предлагаемой работе изучается модель вырожденного релятивистского идеального газа протонов $(p)$, нейтронов $(n)$ и электронов $(e)$ во внешнем постоянном однородном магнитном поле. В рамках этой модели мы исследуем влияние постоянного сверхсильного магнитного поля на термодинамические величины (плотности энергии и числа частищ, давление, намагниченность) вырожденного газа нейтронов, протонов и электронов (nре-газ) с учетом эффектов, обусловленных взаимодействием АММ фермионов с магнитным полем. Некоторые аспекты этой задачи были рассмотрены ранее в работе [19].

Здесь мы изучим некоторые макроскопические эффекты, которые могут иметь место в сильно намагниченной нейтронной звезде. Так, мы покажем, что в сильном магнитном поле с индукцией $B$ все термодинамические величины, характеризуюшие электрически заряженные фермионы, должны испытывать непериодические осцилляции с ростом параметра $|e B|^{-1}$ (e- заряд электрона), которые обусловлены взаимодействием АММ протонов и электронов с магнитным полем, и получим выражения для осциллирующих частей термодинамических величин. Частоты этих осцилляций слабо зависят от величины магнитного поля.

Мы покажем, что уже при относительно невысоких плотностях нуклонов и электронов, зависяших от величины магнитного поля, все фермионы становятся полностью поляризованными по спину и что эффект полной спиновой поляризации нейтронов возникает исключительно вследствие взаимодействия АММ нейтрона с магнитным полем. 
Сформулированы условия, необходимые для возникновения полной спиновой поляризации нейтронов. Показано, что этот эффект оказьвает сушественное влияние на поведение термодинамических величин (плотности энергии и числа частиц, давление, намагниченность) вырожденного газа нейтронов, протонов и электронов в магнитном поле. В частности, эффект полной поляризации спинов нейтронов должен привести к значительному увеличению энергии и давления нейтронного газа. Поэтому полная поляризация спинов нейтронов может оказать сушественное влияние на равновесие экстремально намагниченной нейтронной звезды в отношении гравитационного коллапса и, в частности, может привести к увеличению массы стабильной нейтронной звезды с "вмороженным" магнитным полем по сравнению с массой ненамагниченной звезды при одинаковых плотностях нейтронов.

Нелишне напомнить, что в свое время были предсказаны осцилляционная зависимость намагниченности металлов при низких температурах (эффект де Гааза-ван Альфена), определяемая электронами проводимости в окрестности ферми-поверхности, и осцилляционная зависимость намагниченности свободного электронного газа (осциллящии Ландау) от напряженности магнитного поля [20]-[22]. Осциллящионная зависимость намагниченности электронов при низких температурах является следствием квантования энергии орбитального движения электронов в магнитном поле [20]-[22].

Функции, описываюшие осциллирующие части всех термодинамических величин квантовой системы электрически заряженных фермионов, имеют одну и ту же частоту, а их период по переменной $|e B|^{-1}$ есть постоянная величина, не зависяшая ни от температуры, ни от напряженности магнитного поля. Поведение релятивистского электрон-позитронного газа в постоянном и однородном магнитном поле (в частности, осцилляционная зависимость намагниченности электрон-позитронного газа) в случае $(3+1)$-мерной квантовой электродинамики были изучены в работах [23], [24].

Заметим, что непериодические по $|e B|^{-1}$ осцилляции намагниченности совсем недавно были обнаружены в вырожденном магнитном полупроводнике $\mathrm{HgCr}_{2} \mathrm{Se}_{4}$ [25]. Tepмодинамические и некоторые динамические величины известной модели Намбу-ИонаЛазинио при ненулевых химическом потенциале и внешнем магнитном поле также могут испытывать непериодические магнитные осцилляции, что было показано теоретически в работе [26]. Однако, так как лагранжиан этой модели не содержит параметра массы, фермион должен сначала приобрести массу динамически за счет радиационных эффектов в присутствии внешнего магнитного поля. Следует также отметить, что поскольку модель Намбу-Иона-Лазинио неперенормируема в $3+1$ измерениях, то масса фермиона (а следовательно, и период магнитных осцилляций) зависит не только от напряженности магнитного поля, но и от параметра обрезания.

Мы используем систему единиц, в которой $c=\hbar=1$.

\section{2. СПЕКТРЫ ЭНЕРГИЙ ФЕРМИОНОВ В ПОСТОЯННОМ И ОДНОРОДНОМ МАГНИТНОМ ПОЛЕ}

Собственные значения гамильтониана уравнения Дирака-Паули для протона, обладаюшего AMM, во внешнем постоянном и однородном магнитном поле с учетом эффек- 
тов $\sigma-, \omega-, \rho$-мезонного взаимодействия между нуклонами в приближении внешнего постоянного и однородного (мезонного) поля были получены в работе [16] в виде

$$
E_{p}=\sqrt{p^{2}+\left(\sqrt{|e| B(2 n+1-s \operatorname{sgn} e)+m_{p}^{2}\left(g_{p}^{s} \sigma\right)}-s M_{p} B\right)^{2}}+g_{p}^{o} \omega^{0}-g_{p}^{r} \rho^{0}
$$

где $p$ - проекция импульса протона в направлении внешнего поля $\mathbf{B}, e$ - электрический заряд протона, $n=0,1,2, \ldots$ - главное квантовое число, нумерующее уровни Ландау, $m_{p}\left(g_{p}^{s} \sigma\right)$ - масса протона, в которой учтен вклад от взаимодействия протона со скалярным постоянным полем $\sigma$-мезонов. В тех же внешних полях спектр энергий для нейтрона имеет вид [16]

$$
E_{n}=\sqrt{p^{2}+\left(\sqrt{p_{\perp}^{2}+m_{n}^{2}\left(g_{n}^{s} \sigma\right)}+s M_{n} B\right)^{2}}+g_{n}^{o} \omega^{0}+g_{n}^{r} \rho^{0}
$$

где $p$ и $p_{\perp}-$ компоненты импульса соответственно по и перпендикулярно направлению внешнего магнитного поля $\mathbf{B} ; m_{n}\left(g_{n}^{s} \sigma\right)$ - масса нейтрона, в которой учтен вклад от взаимодействия нейтрона с полем $\sigma$-мезонов; $M_{p, n}-$ АММ протона и нейтрона, соответственно; $s= \pm 1$ - квантовое число, характеризующее проекцию спина фермиона на направление внешнего магнитного поля; $g_{p, n}^{s}, g_{p, n}^{o}$ и $g_{p, n}^{r}$ - константы сильного взаимодействия протонов и нейтронов с классическими постоянными полями $\sigma-, \omega$ - и $\rho$-мезонов, соответственно. Отметим, что взаимодействие нейтрона, обладающего AMM, с классическим электромагнитным полем соответствует неминимальной связи.

Спектр энергий уравнения Дирака-Паули для электрона с учетом АММ во внешнем постоянном и однородном магнитном поле имеет вид [27]

$$
E_{e}=\sqrt{p^{2}+\left(\sqrt{|e| B(2 n+1-s \operatorname{sgn} e)+m_{e}^{2}}-s M_{e} B\right)^{2}},
$$

где $p$ - проекция импульса электрона в направлении внешнего поля, $e, m_{e}$ и $M_{e}-$ электрический заряд, масса и АММ электрона, соответственно. Напомним также значения напряженностей так называемых критических магнитных полей: $B_{0}=m_{e}^{2} /|e|=4.4 \times$ $10^{13}$ Гс для электрона и $B_{0}^{*}=m_{p}^{2} / e=3.4 \cdot 10^{6} B_{0}\left(m_{p}\right.$ и $e-$ масса и заряд протона $)$ для протона. Расстояния между уровнями Ландау в магнитных полях $B_{0}$ для электрона и $B_{0}^{*}$ для протона становятся равными энергиям покоя этих частиц.

Заметим, что $\omega$ и $\rho$ являются векторным и изовекторным мезонными полями, соответственно, и что эффективные массы нуклонов содержат два члена - "затравочный” и пропорциональньй скалярному мезонному полю, причем последний член зависит от конкретной модели лагранжиана взаимодействия нуклонов и мезонных полей, например [16]

$$
m_{p}\left(g_{p}^{s} \sigma\right)=m_{p 0}-g_{p}^{s} \sigma, \quad m_{n}\left(g_{n}^{s} \sigma\right)=m_{n 0}-g_{n}^{s} \sigma
$$

или

$$
m_{p}\left(g_{p}^{s} \sigma\right)=\frac{m_{p 0}^{2}}{m_{p 0}+g_{p}^{s} \sigma}, \quad m_{n}\left(g_{n}^{s} \sigma\right)=\frac{m_{n 0}^{2}}{m_{n 0}+g_{n}^{s} \sigma}
$$


Считается, что химическое равновесие в вырожденном газе нуклонов и электронов может поддерживаться так называемыми прямыми урка-процессами [8]-[11]

$$
p+e \rightarrow n+\nu, \quad n \rightarrow p+e+\bar{\nu},
$$

где $\nu$ и $\bar{\nu}$ обозначают нейтрино и антинейтрино, соответственно. В этих процессах сохраняется плотность полного числа барионов $\left(n_{b}=n_{p}+n_{n}\right)$ и выполняется условие электронейтральности системы

$$
n_{p}=n_{e}
$$

Так как число $n_{b}$ остается постоянным, полная плотность внутренней энергии системы $\mathcal{E}$ фактически зависит только от величины $n_{n}$. Химическое равновесие достигается, когда функция $\mathcal{E}$ имеет минимум, а отношение $x=n_{p} / n_{n}$ в значительной мере определяет уравнение состояния вырожденного газа нуклонов и электронов.

Условие равновесия определяется уравнением для химических потенциалов

$$
\mu_{n}^{*}=\mu_{p}^{*}+\mu_{e}
$$

Поскольку предполагается, что нейтрино покидают звезду, их химический потенциал полагается равным нулю.

Поля мезонов $\sigma, \omega, \rho$, заданные в формулах (1) и (2), определяются соответствуюшими статическими полевыми уравнениями для мезонов, которые содержат массы мезонов, потенциал самовзаимодействия (для $\sigma$-мезонов), плотности барионов и нуклон-мезонные константы связи [16]. В состоянии химического равновесия согласованные решения этих полевых уравнений должны определяться с учетом соотношений (6) и (7). Поэтому поля $\sigma, \omega, \rho$ и нуклон-мезонные константы связи зависят от химических потенциалов и концентраций частиц, а в присутствии внешнего магнитного поля - и от его индукции [16].

Поскольку в формулах (1) и (2) $\sigma, \omega, \rho$ - постоянные величины, то учет обмена мезонами между нуклонами в приближении среднего поля приводит к изменению затравочных масс и энергий Ферми нуклонов. Действительно, энергии Ферми нуклонов $E_{p, n}^{\mathrm{F}}$ фиксированы их химическими потенциалами в отсутствие мезонных полей $\left(\mu_{p, n}^{*}\right)$ и потенциальными энергиями нуклонов во внешнем поле мезонов следующим образом:

$$
E_{p, n}^{\mathrm{F}}=\mu_{p, n}^{*}-g_{p, n}^{o} \omega^{0} \pm g_{p, n}^{r} \rho^{0} \equiv \mu_{p, n}
$$

Эффективные массы нуклонов мы будем обозначать просто $m_{p, n}$.

Если $M_{e, p}=0$, то все уровни энергии, описываемые формулами (1), (3), за исключением лишь уровня с $n=0, s=-1$ (для электрона) и уровня с $n=0, s=+1$ (для протона), двукратно вырожденны: совпадают уровни с $n, s=+1$ и $n+1, s=-1$ (для электрона) и уровни с $n, s=-1$ и $n+1, s=+1$ (для протона). Основными состояниями являются состояние с $n=0, s=-1$ для электрона и состояние с $n=0, s=+1$ для протона; проекция спина электрона и протона в этих состояниях на направление 
внешнего магнитного поля может принимать только одно значение $s=-1$ или $s=+1$, соответственно.

Если $M_{e, p} \neq 0$, то основными состояниями являются состояние с $n=0, s=-1$ для электрона и состояние с $n=0, s=1$ для протона, причем теперь все уровни энергии с $n=0,1,2, \ldots$ двукратно расщеплены по спиновому квантовому числу, т.е. во всех состояниях взаимодействие АММ заряженного фермиона с внешним магнитным полем снимает вырождение по направлениям (проекциям) его спина. Из уравнения (2) следует, что если взаимодействие АММ с магнитным полем учтено, то каждый уровень энергии расшепляется на два уровня, явно зависящих от спина (и $\left.M_{n}\right)$.

В сильных магнитных полях $B \gg B_{0}$ вклады от АММ нуклонов должны быть учтены. Действительно, экспериментальные значения АММ нуклонов суть $M_{p}=$ $\left(g_{p} / 2-1\right) M_{N}$ для протона и $M_{n}=g_{n} M_{N} / 2$ для нейтрона, где $M_{N}=e / 2 m_{p 0}$ - ядерный магнетон, а $g_{p}=5.58, g_{n}=-3.82$ - g-факторы Ланде для протона и нейтрона, соответственно. Отметим, что ядерный магнетон содержит “затравочную” (не эффективную) массу протона.

Поскольку АММ нуклонов имеют порядок $M_{N}$, их энергии связи $\left|M_{n, p}\right| B \cong$ $70\left(B / B_{0}^{*}\right)$ МэВ становятся значительными в полях $B \gg B_{0}$, что может привести к изменениям в условии химического равновесия и в энергиях Ферми нуклонов. Для представляющего интерес диапазона энергий Ферми (от нескольких МэВ до десятков МэВ) ясно, что значительные изменения могут иметь место при $B>10^{-2} B_{0}^{*}$. Для электронов это не так. Чтобы понять причину такого различия, вспомним, что АММ электрона имеет динамический источник (обусловленный так называемыми радиационными поправками), а его величина даже в слабых магнитных полях мала [28]: $M_{e}=-\left(g_{e} / 2-1\right) M_{\mathrm{B}} \equiv$ $-\left(e^{2} / 2 \pi\right) M_{\mathrm{B}}$, где $M_{\mathrm{B}}$ - магнетон Бора. При $B \gg B_{0}$ величина АММ электрона существенно зависит от напряженности магнитного поля и стремится к нулю в области $B \gg B_{0}[29]$. Соответственно вклады от АММ электрона очень малы в области $B_{0}^{*} \gg B \gg B_{0}$.

\section{3. ТЕРМОДИНАМИЧЕСКИЕ ВЕЛИЧИНЫ}

Найдем основные термодинамические величины (плотности числа квантовых состояний и энергии, давление, намагниченность) вырожденного идеального релятивистского газа электронов, протонов и нейтронов в постоянном и однородном магнитном поле $B$, используя формулы для спектров энергий фермионов с учетом их АMМ.

Плотность числа квантовых состояний (ПЧКС) $n_{e, p}$ (в отсутствие поля плотность этого числа совпадает с плотностью числа частищ) и плотность энергии вырожденного релятивистского газа электронов или протонов $\mathcal{E}_{e, p}$ во внешнем постоянном однородном магнитном поле определяются формулами

$$
\begin{gathered}
n_{e, p}=\frac{e B}{2 \pi^{2}} \sum_{s= \pm 1} \sum_{n=0}^{n_{\max }} \int_{0}^{p_{e, p}^{\mathrm{F}}} d p \\
\mathcal{E}_{e, p}=\frac{e B}{2 \pi^{2}} \sum_{s= \pm 1} \sum_{n=0}^{n_{\max }} \int_{0}^{p_{e, p}^{\mathrm{F}}} d p \sqrt{p^{2}+\left(\sqrt{2|e B| n+m_{e, p}^{2}}-s M_{e, p} B\right)^{2}}
\end{gathered}
$$


где $p_{e, p}^{\mathrm{F}}$ - импульс Ферми (для уровня Ландау с заданными $n$ и $s$ ) электронов и протонов, соответственно. Проинтегрировав (8) и (9) по $p$, получим

$$
\begin{gathered}
n_{e, p}=\frac{e B m_{e, p}}{4 \pi^{2}} \sum_{s= \pm 1} \sum_{n=0}^{n_{\max }} z_{n}(e, p), \\
\mathcal{E}_{e, p}=\frac{e^{2} B B_{e, p}}{4 \pi^{2}} \sum_{s= \pm 1} \sum_{n=0}^{n_{\max }}\left[z_{n}(e, p) \sqrt{1+z_{n}^{2}(e, p)+b_{e, p} n}+\right. \\
\left.+\left(\sqrt{1+b_{e, p} n} \mp s M_{e, p} B m_{e, p}^{-1}\right)^{2} \ln \frac{z_{n}(e, p)+\sqrt{1+z_{n}^{2}(e, p)+b_{e, p} n}}{\sqrt{1+b_{e, p} n}}\right] .
\end{gathered}
$$

Здесь введены обозначения $B_{e}=B_{0}, B_{p}=B_{0}^{*}$,

$$
\begin{gathered}
z_{n}^{2}(e, p)=\frac{\mu_{e, p}^{2}-\left(\sqrt{2|e B| n+m_{e, p}^{2}}+s M_{e, p} B\right)^{2}}{m_{e, p}^{2}}, \quad b_{e, p} \equiv \frac{2|e B|}{m_{e, p}^{2}} \\
n_{\max }=\left[\frac{\left(\mu_{e, p} \mp s M_{e, p} B\right)^{2}-m_{e, p}^{2}}{2|e B|}\right]
\end{gathered}
$$

Очевидно, суммирование по $n$ проводится до такого целого числа $n_{\max }$ (зависяшего также от $s)$, которое предшествует значению $n$, при котором величина $z_{n}^{2}(e, p)$ становится отрицательной.

Давление вырожденного газа заряженных частиц в отсутствие внешнего поля определяется выражением [21] $P=-\left(\partial \mathcal{E}_{V} / \partial V\right)_{S=0}$, где $V$ - объем, $S$ - энтропия газа. Для газа заряженных частищ во внешнем электромагнитном поле следует использовать иное выражение. Давление вырожденного релятивистского газа электронов или протонов $P_{e, p}$ во внешнем постоянном однородном магнитном поле можно определить формулой

$$
P_{e, p}=\frac{e B}{2 \pi^{2}} \sum_{s= \pm 1} \sum_{n=0}^{n_{\max }} \int_{0}^{p_{e, p}^{\mathrm{F}}} d p \frac{p^{2}}{\sqrt{p^{2}+\left(\sqrt{2|e B| n+m_{e, p}^{2}}+s M_{e, p} B\right)^{2}}}
$$

После интегрирования получим

$$
\begin{aligned}
P_{e, p}= & \frac{e^{2} B B_{e, p}}{4 \pi^{2}} \sum_{s= \pm 1} \sum_{n=1}^{n_{\max }}\left[z_{n}(e, p) \sqrt{1+z_{n}^{2}(e, p)+b_{e, p} n}-\right. \\
& \left.-\left(\sqrt{1+b_{e, p} n} \mp s M_{e, p} B m_{e, p}^{-1}\right)^{2} \ln \frac{z_{n}(e, p)+\sqrt{1+z_{n}^{2}(e, p)+b_{e, p} n}}{\sqrt{1+b_{e, p} n}}\right] .
\end{aligned}
$$

Намагниченность газа определяется формулой

$$
\mathcal{M}_{e, p}=-\frac{\partial \mathcal{E}_{e, p}}{\partial B} .
$$


ПЧКС нейтронов в постоянном магнитном поле можно задать формулой

$$
n_{n}=\frac{1}{2 \pi^{2}} \sum_{s=1,-1} \int_{0}^{p_{\perp}^{\mathrm{F}}} \int_{0}^{p^{\mathrm{F}}} d p_{\perp} d p p_{\perp}
$$

где

$$
p^{\mathrm{F}}=\sqrt{\mu_{n}^{2}-\left(\sqrt{p_{\perp}^{2}+m_{n}^{2}}+s M_{n} B\right)^{2}}, \quad p_{\perp}^{\mathrm{F}}=\sqrt{\left(\mu_{n}-s M_{n} B\right)^{2}-m_{n}^{2}} .
$$

Отметим, что при интегрировании всех выражений по $p$ и $p_{\perp}$ вначале проводится интегрирование по $p$.

Интегрируя (16), найдем

$$
\begin{aligned}
n_{n}= & \frac{1}{2 \pi^{2}} \sum_{s=1,-1}\left\{\frac{\left(\mu_{n}^{2}-m_{n *}^{2}\right)^{3 / 2}}{3}+\right. \\
& \left.+\frac{s M_{n} B}{2}\left[m_{n *} \sqrt{\mu_{n}^{2}-m_{n *}^{2}}-\mu_{n}^{2} \arcsin \frac{\sqrt{\mu_{n}^{2}-m_{n *}^{2}}}{\mu_{n}}\right]\right\}
\end{aligned}
$$

где $m_{n *}=m_{n}+s M_{n} B$. Плотность энергии релятивистского газа нейтронов в постоянном магнитном поле при нулевой температуре $\mathcal{E}_{n}$ определяется формулой

$$
\mathcal{E}_{n}=\frac{1}{2 \pi^{2}} \sum_{s=1,-1} \int_{0}^{p_{\perp}^{\mathrm{F}}} \int_{0}^{p^{\mathrm{F}}} d p_{\perp} d p p_{\perp} \sqrt{p^{2}+\left(\sqrt{p_{\perp}^{2}+m_{n}^{2}}+s M_{n} B\right)^{2}} .
$$

Интегрируя (18), получим

$$
\begin{aligned}
\mathcal{E}_{n}= & \frac{1}{16 \pi^{2}} \sum_{s=1,-1}\left\{\mu_{n}\left(\mu_{n}^{2}-m_{n *}^{2}\right)^{3 / 2}+\mu_{n}^{3} \sqrt{\mu_{n}^{2}-m_{n *}^{2}}-\frac{m_{n *}^{4}}{2} \ln \frac{\mu_{n}+\sqrt{\mu_{n}^{2}-m_{n *}^{2}}}{\mu_{n}-\sqrt{\mu_{n}^{2}-m_{n *}^{2}}}-\right. \\
& -\frac{s M_{n} B}{3}\left[-5 m_{n *} \mu_{n} \sqrt{\mu_{n}^{2}-m_{n *}^{2}}+\right. \\
& \left.\left.+2 m_{n *}^{3} \ln \frac{\mu_{n}+\sqrt{\mu_{n}^{2}-m_{n *}^{2}}}{\mu_{n}-\sqrt{\mu_{n}^{2}-m_{n *}^{2}}}+\mu_{n}^{3} \arcsin \frac{\sqrt{\mu_{n}^{2}-m_{n *}^{2}}}{\mu_{n}}\right]\right\} .
\end{aligned}
$$

Давление сильно намагниченного нейтронного газа при нулевой температуре можно определить формулой $P_{n}=-\left(\partial \mathcal{E}_{n} V / \partial V\right)_{S=0}$, что дает

$$
\begin{aligned}
P_{n}= & \frac{1}{8 \pi^{2}} \sum_{s=1,-1}\left\{\left(\frac{\mu_{n}^{3}}{3}-\frac{5 \mu_{n} m_{n *}^{2}}{6}\right) \sqrt{\mu_{n}^{2}-m_{n *}^{2}}+\frac{m_{n *}^{4}}{4} \ln \frac{\mu_{n}+\sqrt{\mu_{n}^{2}-m_{n *}^{2}}}{\mu_{n}-\sqrt{\mu_{n}^{2}-m_{n *}^{2}}}+\right. \\
& +s M_{n} B\left[\left(\frac{m_{n *}^{3}}{2 \mu_{n}}-\frac{25 m_{n *} \mu_{n}}{18}\right) \sqrt{\mu_{n}^{2}-m_{n *}^{2}}+\frac{m_{n *}\left(\mu_{n}^{2}-m_{n *}^{2}\right)}{18 \mu_{n}}+\right. \\
& \left.\left.+\frac{m_{n *}^{3}}{3} \ln \frac{\mu_{n}+\sqrt{\mu_{n}^{2}-m_{n *}^{2}}}{\mu_{n}-\sqrt{\mu_{n}^{2}-m_{n *}^{2}}}+\frac{\mu_{n} m_{n *}^{2}}{6} \arcsin \frac{\sqrt{\mu_{n}^{2}-m_{n *}^{2}}}{\mu_{n}}\right]\right\} .
\end{aligned}
$$


Для намагниченности газа нейтронов нетрудно получить выражение

$$
\begin{aligned}
\mathcal{M}_{n}= & \frac{1}{4 \pi^{2}} \sum_{s=1,-1} s M_{n}\left[\mu_{n} \sqrt{\mu_{n}^{2}-m_{n *}^{2}}\left(\frac{7 m_{n}}{6}+s M_{n} B\right)+\right. \\
& \left.+\frac{4}{3} m_{n *}^{3} \ln \frac{\mu_{n}+\sqrt{\mu_{n}^{2}-m_{n *}^{2}}}{\mu_{n}-\sqrt{\mu_{n}^{2}-m_{n *}^{2}}}+\mu_{n}^{3} \arcsin \frac{\sqrt{\mu_{n}^{2}-m_{n *}^{2}}}{\mu_{n}}\right] .
\end{aligned}
$$

Из этой формулы для намагниченности нейтронного газа в двух предельных случаях имеем

$$
\mathcal{M}_{n} \approx \begin{cases}\frac{9 e^{2} B m_{n}}{2 \pi^{2} m_{n 0}}\left(\frac{n_{n}}{n_{0 n}}\right)^{1 / 3}, & n_{n} \ll n_{0 n}, \\ \frac{9 e^{2} B}{2 \pi^{2}}\left(\frac{n_{n}}{n_{0 n}}\right)^{2 / 3}, & n_{n} \gg n_{0 n}, \quad n_{0 n}=\frac{m_{n 0}^{3}}{3 \pi^{2}} .\end{cases}
$$

Монотонные части термодинамических величин намагниченности протонов определяются формулами (19)-(21) с $\mu_{p}, m_{p}, M_{p}$ и $n_{p} / n_{0 p}$ вместо $\mu_{n}, m_{n}, M_{n}$ и $n_{n} / n_{0 n}$, соответственно. Например, монотонные части намагниченности протонов после этих замен будут определяться в аналогичных предельных случаях формулой (22), в которой численный коэффициент 9 и отношение $n_{n} / n_{0 n}$ следует заменить на 13 и $n_{p} / n_{0 p}$, соответственно. Обратим внимание на то, что монотонные части намагниченностей вырожденных нейтронов и протонов при относительно невысоких плотностях содержат множители $m_{n, p} / m_{n 0, p 0}$, в числители которых входят эффективные массы нуклонов. А так как для любых моделей, учитывающих нуклон-нуклонное взаимодействие в приближении среднего поля, эффективные массы нуклонов меньше их “затравочных" масс, то уменьшаются и монотонные части намагниченностей вырожденных нейтронов и протонов в таких моделях.

\section{4. МАГНИТНЫЕ ОСЦИЛЛЯЦИИ И ЭФФЕКТЫ АНИЗОТРОПИИ}

Все полученные вьше точные выражения для термодинамических величин заряженных фермионов, обладаюших АММ, содержат осциллируюшие члены (осцилляции Ландау). Чтобы это показать, удобно ввести в соответствуюшие выражения $\theta$-функции аргумента

$$
x \equiv z_{n}^{2}(e, p) \cong \frac{\mu_{e, p}^{2}-2|e B| n-m_{e, p}^{2}-2 s m_{e, p} M_{e, p} B}{m_{e, p}^{2}}
$$

и распространить суммирование по $n$ от 0 до $\infty$. Тогда осциллирующие функции можно выделить явно, используя для суммирования по $n$ известную формулу суммирования Пуассона [26]

$$
\sum_{n=0}^{\infty} f(n)=\int_{0}^{\infty} f(x) d x+\frac{f(0)}{2}+2 \sum_{k=1}^{\infty} \int_{0}^{\infty} f(x) \cos (2 \pi k x) d x
$$


В результате после некоторых преобразований получим, например, для основного члена осциллируюшей части плотности энергии газа заряженных фермионов при нулевой температуре выражение

$$
\mathcal{E}_{\mathrm{osc}} \cong \frac{\mu_{e, p}}{16 \pi^{5 / 2}} \sum_{s= \pm 1} \sum_{k=1}^{\infty}\left(\frac{|e B|}{\pi k}\right)^{3 / 2} \frac{\cos (2 \pi k \omega-\pi / 4)}{k \omega},
$$

где $\omega=\left(\mu_{e, p}^{2}-m_{e, p}^{* 2}\right) /(2|e B|), m_{e, p}^{*} \cong m_{e, p} \pm M_{e, p} B$.

По переменной $(2|e B|)^{-1}$ плотность энергии газа заряженных фермионов осциллирует с частотами

$$
\omega_{ \pm}=\mu_{e, p}^{2}-\left(m_{e, p} \pm M_{e, p} B\right)^{2}
$$

которые, как видно из последней формулы, зависят от магнитной индукции $B$. Заметим, что с такими же частотами осциллируют плотность числа частиц, давление и намагниченность газа заряженных фермионов. Мы видим, что магнитные осцилляции всех физических величин, которые описывают поведение газа заряженных фермионов, обладающих АMМ, не являются строго периодическими.

Следует подчеркнуть, что осциллирующие части плотности энергии и давления $n p e-$ газа малы по сравнению с их монотонными частями и тем более по сравнению с их значениями в отсутствие поля. Что касается осциллируюшей части намагниченности электрически заряженных фермионов, то она, наоборот, велика по сравнению с ее монотонной частью при определенном химическом потенциале (или заданной плотности заряженных фермионов). Так, например, абсолютные величины осциллирующей и монотонной частей намагниченности приближенно соотносятся как

$$
\mathcal{M}_{\mathrm{osc}} \sim \mathcal{M}_{\mathrm{mon}}\left(\frac{\mu}{M B}\right)^{1 / 2}
$$

где $\mu$ - химический потенциал, а $M$ - магнитньй момент заряженного фермиона. Используя соотношение (25), величину осциллирующей части намагниченности газа протонов можно оценить как

$$
\mathcal{M}_{\mathrm{osc}} \sim \begin{cases}e^{2}\left(B B_{0}^{*}\right)^{1 / 2} \frac{m_{p}}{m_{p 0}}\left(\frac{n_{p}}{n_{0 n}}\right)^{2 / 3}, & n_{p} \ll n_{0 n}, \\ e^{2}\left(B B_{0}^{*}\right)^{1 / 2}\left(\frac{n}{n_{0 n}}\right)^{5 / 6}, & n_{p} \gg n_{0 n} .\end{cases}
$$

При плотности числа протонов, удовлетворяющей условию $n_{p} \ll n_{0 n}$, осциллирующая часть намагниченности пре-газа может оказывать существенное влияние на поведение макроскопической напряженности магнитного поля

$$
\mathbf{H}=\mathbf{B}-4 \pi \mathcal{M}(\mathbf{B})
$$

Известно [30], что в металлах в такой ситуации осцилляции намагниченности могли бы привести к появлению на кривой зависимости макроскопической напряженности $H$ от 
индукции $B$ ряда последовательных перегибов. В действительности появление перегибов на обсуждаемой кривой приводит к последовательным фазовым переходам в намагниченном теле со скачкообразными изменениями индукции [30].

В работе [13] было показано, что в магнитном поле с $B_{0}^{*} \gg B \gg B_{0}$ равновесная плотность протонов увеличивается по сравнению со случаем $B=0$ во всем интервале изменения плотности нейтронов, оставаясь тем не менее сушественно меньше $n_{n}$ во всем интервале изменения плотности нейтронов. Поэтому основной вклад в эффекты, которые могут иметь место в сильно намагниченной нейтронной звезде, дают нейтроны.

При сверхвысоких плотностях $n_{n} \gg n_{0 n}$ такие эффекты могли бы оказаться весьма значительными. Следует, конечно, иметь в виду, что уже при плотностях порядка плотности вешества ядер становится сушественным сильное взаимодействие между нуклонами, так что в этой области значений полученные выше формулы могут иметь лишь качественный смысл. Тем не менее, если предположить, что, например, формула для намагниченности нейтронного газа качественно верна, мы придем к выводу о возможности возникновения спонтанной намагниченности в вырожденном сверхплотном нейтронном газе. Действительно, подставляя в уравнение (27) выражение для намагниченности (22), нетрудно показать, что уравнение (27) может иметь формальное ненулевое решение $(B \neq 0)$ при $\mathbf{H} \rightarrow 0$, только если в области значений $n_{n} \gg n_{0 n}$ выполняется соотношение

$$
\left(\frac{n}{n_{0 n}}\right)^{2 / 3}=\frac{\pi}{18 e^{2}} \cong 24 \text {. }
$$

Величина магнитной индукции должна быть ограничена, например, условием малости осциллирующей части намагниченности протонов по сравнению с намагниченностью нейтронов $\mathcal{M}_{\text {osc }}<\mathcal{M}_{n}$, что дает

$$
\frac{B}{B_{0}^{*}}>2\left(\frac{n_{p}}{n_{0 n}}\right)^{5 / 3}\left(\frac{n_{0 n}}{n_{n}}\right)^{4 / 3} \sim 3.2 \cdot 10^{-3}\left(\frac{n_{p}}{n_{0 n}}\right)^{5 / 3},
$$

или условием стабильности нейтронной звезды, вытекающим из теоремы вириала.

Из формул (1), (2) следует, что в моделях, учитывающих нуклон-нуклонные взаимодействия, эффективные массы нуклонов становятся меньше их "затравочных" масс; уменьшаются и энергии Ферми нуклонов.

Рассмотрим полный тензор напряжений вешества и электромагнитного поля в нашей модели. Максвелловский тензор напряжений однородного магнитного поля имеет следующие ненулевые компоненты:

$$
T^{00}=-T^{33}=T^{11}=T^{22}=\frac{H B}{8 \pi} .
$$

Основной вклад в давление вещества в центральной области сильно намагниченной нейтронной звезды дают вырожденные нейтроны в магнитном поле; давление вырожденного газа нейтронов описывается формулой (20). Главные члены компонент полного

5 Теоретическая и математическая физика, т. 133, № 1, 2002 г. 
тензора напряжений в постоянном однородном магнитном поле можно представить в виде [19]

$$
T_{11}=T_{22}=P_{\perp}+\left(\frac{1}{2}-\gamma\right) \mathcal{M} B+\frac{B^{2}}{8 \pi}, \quad T_{33}=P_{3}+\left(\frac{3}{2}-\gamma\right) \mathcal{M} B-\frac{B^{2}}{8 \pi}
$$

где $P_{\perp}=P_{3} \equiv P$ - давление газа в отсутствие магнитного поля, а $\gamma=5 / 3,4 / 3$ при $n_{n} \ll n_{0 n}, n_{n} \gg n_{0 n}$, соответственно. Выражение $\mathcal{M} B$ представляет собой плотность магнитной энергии нейтронов. При $n_{n} \ll n_{0 n}$ и $n_{n} \gg n_{0 n}$ компоненты полного тензора напряжений можно также представить в виде

$$
\begin{aligned}
& T_{11}=T_{22}=(\gamma-1) \mathcal{E}_{\gamma}+\left(\frac{1}{2}-\gamma\right) \mathcal{M} B+\frac{B^{2}}{8 \pi}, \\
& T_{33}=(\gamma-1) \mathcal{E}_{\gamma}+\left(\frac{3}{2}-\gamma\right) \mathcal{M} B-\frac{B^{2}}{8 \pi}
\end{aligned}
$$

где

$$
\mathcal{E}_{\gamma=5 / 3} \cong \frac{3 m_{n}^{4}}{24 \pi^{2}}\left(\frac{n_{n}}{n_{0 n}}\right)^{5 / 3}, \quad \mathcal{E}_{\gamma=4 / 3} \cong \frac{3 m_{n}^{4}}{45 \pi^{4}\left(3 \pi^{2}\right)^{2 / 3}}\left(\frac{n_{n}}{n_{0 n}}\right)^{4 / 3}
$$

- плотность энергии при малой и большой плотностях нейтронов, соответственно. Мы также полагаем, что химический потенциал газа связан с плотностью $n_{n}$ соотношением $3 \pi^{2} n_{n}=\left(\mu_{n}^{2}-m_{n *}^{2}\right)^{3 / 2}$.

Плотность магнитной энергии нейтронов при $n_{n} \ll n_{0 n}$ можно оценить формулой

$$
\mathcal{M} B \simeq 2 \cdot 10^{4} \mathcal{E}_{\gamma=5 / 3}\left(\frac{B}{B_{0}^{*}}\right)^{2}\left(\frac{n_{0 n}}{n_{n}}\right)^{4 / 3}
$$

Осциллируюшая часть макроскопической напряженности дает основной вклад в давление, возникающее вследствие намагниченности $n p e$-газа в магнитном поле. Этот вклад может оказываться достаточно существенным даже в центральной области нейтронной звезды, где при химическом равновесии плотность нейтронов более чем на порядок может превьшать плотность протонов. Видно, что в обсуждаемой нами конфигурации магнитного поля полный тензор напряжений анизотропен.

Оценки различных вкладов в полный тензор напряжений показывают, что вклад от магнитного поля в полное давление может доминировать в поле с $B>10^{-2} B_{0}^{*}$ при плотностях $n_{n} \leqslant n_{0 n}$, которые достигаются лишь в центральной области нейтронной звезды. Мы видим, что эффекты, обусловленные взаимодействием АММ нуклонов с сверхсильным магнитным полем, весьма значительны, так что полное описание состояния вешества в намагниченной нейтронной звезде с необходимостью требует учета этих эффектов. 


\section{5. ЭФФЕКТЫ, ОБУСЛОВЛЕННЫЕ ПОЛЯРИЗАЦИЕЙ СПИНОВ}

Из решений уравнения Дирака и выражения для спектра энергий электрона (без учета AMM) в постоянном и однородном магнитном поле следует (см., например, [29]), что спин электрона в основном состоянии может быть ориентирован только против направления магнитного поля. Это означает, что вырожденный газ электронов при плотности $n_{e}$, удовлетворяюшей соотношению

$$
\frac{\sqrt{2} n_{e}}{3 n_{0 n}}\left(\frac{B_{0}^{*}}{B}\right)^{3 / 2}<1,
$$

в основном состоянии в однородном магнитном поле с $B_{0}^{*} \gg B \gg B_{0}$ должен быть полностью поляризованным. По той же причине возможна полная спиновая поляризация протонов в постоянном однородном магнитном поле с $B_{0}^{*} \gg B \gg B_{0}$, даже если взаимодействие АММ протона с магнитным полем не учитывается. Это возможно при относительно низких плотностях протонов, когда все протоны смогут находиться на низшем уровне Ландау $n=0$; их спины должны быть направлены по вектору магнитной индукции, т.е. $s=1$.

Полностью поляризованным в присутствии постоянного и однородного магнитного поля должен стать и рассматриваемый нами вырожденный газ нуклонов и электронов, химическое равновесие в котором поддерживается вышеупомянутыми урка-процессами. Действительно, так как энергия нейтрона (а значит, и внутренняя энергия) явно зависит от проекции спина на направление магнитного поля, устойчивыми состояниями нейтронов в постоянном и однородном магнитном поле будут состояния с одним (определенным) значением проекций спинов нейтронов.

Рассмотрим поведение газа нейтронов, считая, что магнитное поле изменяется адиабатически. Будем также считать, что полное число нейтронов равно $n$ (здесь и ниже индекс $n$ в выражении для плотности нейтронов $n_{n}$ опускаем), а значит, и их химический потенциал в отсутствие магнитного поля $\mu_{n}$ фиксированы. В момент включения магнитного поля плотности нейтронов с проекциями спинов по и против направления поля равны $n / 2$. С ростом магнитного поля изменяются ПЧКС нейтронов со спинами, направленными по и против поля, за счет перераспределения нейтронов по спиновым состояниям.

Можно выделить три характерных значения магнитной индукции, зависящих от химического потенциала или от полного числа нейтронов. Так, в постоянном однородном магнитном поле с $B_{0}^{*} \gg B \gg B_{0}$ сумма максимальной кинетической и потенциальной энергии нейтрона, обладаюшего АММ, со спином $s=1$ будет равна минимальной энергии нейтрона со спином $s=-1$ при значении магнитной индукции, определяемом формулой

$$
\frac{\mu_{n}^{2}-\left(m_{n}+M_{n} B\right)^{2}}{2 m_{n}}+M_{n} B=-M_{n} B,
$$

из которой, пренебрегая малым членом $\left(M_{n} B\right)^{2}$, найдем

$$
B_{1}=\frac{\mu_{n}^{2}-m_{n}^{2}}{4\left|M_{n}\right| m_{n}}
$$


Так как нейтроны нерелятивистские и $M_{n}<0$, то

или по-другому

$$
\left|M_{n}\right| B=\frac{\mu_{n}^{2}-\left(m_{n}+M_{n} B\right)^{2}}{4 m_{n}} \simeq \frac{\left(6 \pi^{2} n\right)^{2 / 3}}{4 m_{n}}
$$

$$
\left(\frac{n}{n_{0 n}}\right)^{2 / 3} \simeq \frac{2^{1 / 3}\left|M_{n}\right| B}{m_{n}} \simeq 2^{1 / 3} \frac{B m_{n 0}}{B_{0}^{*} m_{n}} .
$$

Для полной поляризации нейтронов плотности $n$ в магнитном поле необходимо, чтобы ПЧКС нейтронов с $s=1$ стала бы равной $n$ (ср. с [16]).

Учитывая, что в момент включения магнитного поля плотности нейтронов с проекциями спинов по и против направления поля равны $n / 2$, из формулы (17) получаем, что в магнитном поле с индукцией $B_{2}=x\left(\mu_{n}^{2}-m_{n}^{2}\right) /\left(2\left|M_{n}\right|\right)$ ПЧКС нейтронов с $s=1$ будет равна $n$, если выполняется равенство $2=(1+x)^{3 / 2}$, откуда находим $x \simeq 0.6$. Заметим, что $B_{2}>B_{1}$. Например, если $m_{n} \sim m_{n 0}$, то при ядерных плотностях нейтроны могут стать полностью поляризованными по спину в магнитном поле $B \simeq 10^{-2} B_{0}^{*}$. Аналогично можно было бы прийти к выводу о возможности полной спиновой поляризации протонов в постоянном однородном магнитном поле с $B_{0}^{*} \gg B \gg B_{0}$ за счет взаимодействия АММ протона с магнитным полем.

Полная поляризация спинов вырожденных нейтронов в сверхсильном магнитном поле должна приводить к увеличению давления вещества и вследствие этого к более “жесткому" уравнению состояния [16]. Это можно понять, если рассматривать плотность числа нейтронов, определяемую формулой (17), как функцию магнитной индукции $B$. Действительно, при увеличении магнитной индукции увеличивается ПЧКС нейтронов в спин-поляризованном состоянии $s=1$, что видно из формулы (17). Можно ввести понятие эффективного импульса Ферми вырожденных спин-поляризованных нейтронов в присутствии магнитного поля, который с ростом поля должен увеличиваться. Это, в свою очередь, должно привести к соответствуюшему увеличению давления вещества за счет вырожденных поляризованных по спину нейтронов. При увеличении магнитного поля должны увеличиваться и другие термодинамические величины, характеризующие нейтронный газ. Если энергия Ферми и, следовательно, плотность числа нейтронов остаются неизменными, то увеличение термодинамических величин, которые характеризуют вырожденный газ нейтронов, может происходить только за счет увеличения ПЧКС нейтронов в присутствии магнитного поля и перераспределения нейтронов по спиновым состояниям.

Когда магнитная индукция достигнет значения (зависящего от полного числа нейтронов) $B \simeq\left(\mu_{n}^{2}-m_{n}^{2}\right) /\left(2\left|M_{n}\right|\right)$, ПЧКС нейтронов с проекцией спина на направление магнитного поля $s=1$ примет максимальное значение $\sqrt{2} n$. Действительно, вклад в ПЧКС нейтронов со спином $s=1$ дают состояния с

$$
\sqrt{\mu_{n}^{2}-\left(m_{n}-\left|M_{n}\right| B\right)^{2}} \geqslant 0
$$

а вклад в ПЧКС нейтронов со спином $s=-1-$ состояния с

$$
\sqrt{\mu_{n}^{2}-\left(m_{n}+\left|M_{n}\right| B\right)^{2}} \geqslant 0 .
$$


Знак равенства в последнем соотношении дает точное уравнение для определения магнитной индукции, при которой ПЧКС нейтронов со спином, направленным против поля $(s=-1)$, обрашается в нуль. Интересуюшее нас решение этого уравнения, очевидно, есть

$$
\left|M_{n}\right| B=-m_{n}+\mu_{n} .
$$

При значении магнитной индукции $B_{3}=\left(\mu_{n}-m_{n}\right) /\left|M_{n}\right|$ область значений энергии, которая дает вклад в ПЧКС нейтронов со спином по направлению магнитного поля $(s=1)$, становится максимальной и равной $\sqrt{\mu_{n}^{2}-\left(\mu_{n}-2 m_{n}\right)^{2}}=\sqrt{4 m_{n}\left(\mu_{n}-m_{n}\right)}$. Следовательно, если плотность числа нейтронов в отсутствие магнитного поля была равна $n$, то в магнитном поле с индукцией $B_{3}$ вакантными останутся $(\sqrt{2}-1) n$ состояний со спином $s=1$. Заметим, что $B_{3}>B_{2}>B_{1}$. В слабом магнитном поле $\mu_{n}-$ $m_{n} \gg\left|M_{n}\right| B$ (в нерелятивистском пределе) вклад в плотность числа нейтронов с любой проекцией спина дает область значений энергии $\sqrt{\mu_{n}^{2}-m_{n}^{2}} \cong \sqrt{2 m_{n}\left(\mu_{n}-m_{n}\right)}$.

Вклады в плотности энергии, числа частиц и давление (см. формулы $(17),(19),(20))$ дает только член с $s=1$. При этом основной вклад дают члены, не зависяшие от магнитной индукции. Когда магнитная индукция достигнет значения, при котором все нейтроны будут находиться в спин-поляризованном состоянии $s=1$, увеличение значений всех термодинамических величин вешества должно прекратиться. При дальнейшем увеличении магнитного поля термодинамические величины должны уменьшаться за счет возрастания вклада членов в выражениях $(17),(19),(20)$, зависящих от поля, причем, пока значения магнитной индукции удовлетворяют неравенству $B \ll B_{0}^{*}$, это уменьшение не должно быть сколько-нибудь сушественным.

Нетрудно показать, что плотность энергии и давление нейтронов при достижении магнитной индукцией значения $B=\left(\mu_{n}-m_{n}\right) /\left|M_{n}\right|$ могли бы максимально увеличиться примерно в $2 \sqrt{2}$ раза, а плотность числа частиц - примерно в $\sqrt{2}$ раза по сравнению со значениями этих величин в отсутствие магнитного поля. Это могло бы произойти за счет увеличения ПЧКС нейтронов при условии, что нейтронов достаточно для того, чтобы заполнить все новые квантовые состояния.

Отметим, что полная спиновая поляризация в вырожденном газе нейтронов возможна при относительно невысоких плотностях, т.е. при нерелятивистских значениях энергии Ферми, так что $\mu_{n} \sim m_{n}^{*}$. Будем также полагать, что $m_{n} \gg\left|M_{n}\right| B$. При этих условиях формулу (17) для ПЧКС спин-поляризованных нейтронов можно представить в виде

$$
n_{n}=\frac{1}{2 \pi^{2}} \sum_{s=1,-1} \frac{\left(\mu_{n}^{2}-m_{n *}^{2}\right)^{3 / 2}}{3}\left(1-\frac{3 s M_{n} B}{4 m_{n}^{*}}\right) .
$$

Из условия $n_{n}>0$ следует, что должны выполняться неравенства $\mu_{n}-m_{n}-\left|M_{n}\right| B>0$ и $\mu_{n}-m_{n}+\left|M_{n}\right| B>0$. При $B=\left(\mu_{n}-m_{n}\right) /\left|M_{n}\right|$ вклад в $n_{n}$ дают только состояния c $s=1$. При этом эффективный импульс Ферми вырожденных нейтронов увеличится в $\sqrt{2}$ раза, что и даст увеличение плотности числа частиц в $\sqrt{2}$ раза по сравнению с ее значением в отсутствие магнитного поля.

Намагниченность нейтронов, которую можно определить как разность чисел нейтронов со спинами по полю $\left(n_{+}\right)$и против поля $\left(n_{-}\right)$, умноженную на АММ нейтрона, 
$\mathcal{M}_{n}=M_{n}\left(n_{+}-n_{-}\right)$, достигает максимального значения $\mathcal{M}_{\max }=M_{n} n_{+}$при полной спиновой поляризации нейтронов.

\section{6. ЗАКЛЮЧЕНИЕ}

Выше показано, что эффект полной поляризации спинов вырожденных нейтронов в сверхсильном магнитном поле приводит к значительному увеличению давления вещества и вследствие этого он может существенно изменить условия равновесия экстремально намагниченной нейтронной звезды в отношении ее возможного гравитационного коллапса. В конечном итоге этот эффект должен привести к увеличению массы стабильной намагниченной нейтронной звезды.

К новым интересным следствиям может привести учет квантовых обменных взаимодействий нейтронов. Так, если допустить, что квантовые обменные взаимодействия проявятся и могут стать существенными в достаточно плотном нейтронном газе, то можно ожидать возникновения спонтанной намагниченности в вырожденном плотном (но, что важно, не сверхплотном) нейтронном газе. Возможность этого эффекта была оценена в работе [31] в предположении, что обменное взаимодействие в вырожденном нерелятивистском газе нейтронов можно описать феноменологически аналогично описанию обменного взаимодействия электронов проводимости в металлах [32]. С учетом такого (ферромагнитного) обменного взаимодействия при определенных условиях, как известно [32], ферромагнитное состояние будет иметь меньшую энергию, чем парамагнитное. Как и для системы электронов, если энергия обменного взаимодействия в этом случае будет зависеть от того, насколько перекрываются волновые функции нейтронов, то появления неустойчивости плотности энергии нейтронов в парамагнитном состоянии можно ожидать в достаточно плотном газе [31]. Оценка минимального числа нейтронов $N=n_{n} V$ в объеме $V$, при котором переход из парамагнитного состояния в ферромагнитное может стать возможным, получена в работе [31],

$$
N>\frac{2}{\epsilon g^{2}}\left(\frac{n_{n}}{n_{0 n}}\right)^{1 / 3} \exp \left[c\left(\frac{n_{0 n}}{n_{n}}\right)^{1 / 3}\right],
$$

где $\epsilon$ - малая постоянная, $g$ - константа сильного взаимодействия, $c$ - постоянная порядка 1. Для нейтронных плотностей, представляющих интерес, на основании этой оценки можно предположить, что центральная область нейтронной звезды состоит из ферромагнитных (нейтронных) доменов.

Приближение однородного магнитного поля нуждается в некотором уточнении. Действительно, при относительно низких плотностях частиц, т.е. в нерелятивистском случае, однородным следует считать поле, изменением которого можно пренебречь на расстояниях порядка $\hbar / p^{\mathrm{F}}$. Поэтому локально магнитное поле в нейтронной звезде является аксиально-симметричным и слабо неоднородным. Точные решения и спектр энергий нейтрона известны только для случая внешнего аксиально-симметричного магнитного поля, вектор напряженности которого (в цилиндрических координатах $r, \varphi, z)$ имеет вид

$$
\mathbf{B}=\left(0,0, \frac{a}{r}+B\right)
$$


Собственная функция уравнения Дирака-Паули в этом поле определяется следующими собственными значениями (интегралами движения): энергией $E$, проекцией импульса на направление поля (ось $z$ ) $p$, проекцией полного момента на это направление $l+1 / 2$ и спиновым квантовым числом $s$, описывающим проекцию спина нейтрона на направление магнитного поля. Оказывается, что все состояния нейтрона с проекцией спина на направление поля $(s=1)$ принадлежат к состояниям с квазинепрерывным спектром энергий, а все состояния нейтрона с проекцией спина против поля $(s=-1)-$ к состояниям с непрерывным спектром энергий.

Квазинепрерывный спектр энергий нейтрона в магнитном поле (39) найден в работе [33],

$$
E_{p, n, l, s=1}=\sqrt{p^{2}+\left(E_{n, l}-\left|M_{n}\right| B\right)^{2}}
$$

c

$$
E_{n, l}=m_{n}\left[1+\frac{a^{2}}{\left(n+\sqrt{(l+1 / 2)^{2}-a^{2}}\right)^{2}}\right]^{-1 / 2}<m_{n}
$$

где $a=M_{n} B / m_{n}$ - безразмерная величина. Очевидно, при $\left|M_{n} B\right| \ll m_{n}$ кратность вырождения квазинепрерьвного спектра энергий нейтрона по $l$ равна $n-1$. Поэтому, если в центральной области нейтронной звезды магнитное поле имеет вид (39), то значительная часть нейтронов может заполнить состояния, принадлежащие квазидискретным уровням энергии (40), в которых нейтроны будут полностью поляризованы по спину.

Известно, что эффекты квантования энергии и орбит поперечного (вектору напряженности поля) движения заряженных частиц в постоянном и однородном магнитном поле приводят к увеличению равновесной плотности протонов, “смягчению" уравнения состояния пре-газа, т.е. к уменьшению давления вешества и, в конечном итоге, к изменению соотношения между массой и радиусом намагниченной нейтронной звезды по сравнению со случаем нейтронной звезды без “вмороженного" магнитного поля. Квантование "поперечной" энергии нейтронов в рассматриваемом постоянном неоднородном магнитном поле, описываемое формулой (40), также может привести к “смягчению” уравнения состояния пре-газа и уменьшению давления вещества. Следует только подчеркнуть, что это уменьшение может стать весьма сушественным, поскольку в центральной области нейтронной звезды плотность протонов составляет всего $0.1 n_{n}$, так что основной вклад в давление вещества дают нейтроны.

\section{Список литературы}

[1] B. Paczynski. Acta Astron. 1992. V. 42. P. 145; C. Thompson, R. C. Duncan. Astrophys. J. 1996. V. 473. P. 322.

[2] R. C. Duncan. Physics in ultra-strong magnetic fields. astro-ph/0002442.

[3] C. Kouveliotou et al. Nature. 1998. V. 393. P. 235.

[4] K. Hurley et al. Nature. 1999. V. 397. P. 41.

[5] C. ШШапиро, С. Тьюколски. Черные дыры, белые карлики и нейтронные звезды. М.: Мир, 1985.

[6] S. Glosh et al. Electrical conductivity at the core of a magnetar. astro-ph/0106153. 
[7] D. Lai, S. Shapiro. Astrophys. J. 1991. V. 383. P. 745.

[8] R. Kippenhahn, A. Weigert. Stellar Structure and Evolution. Berlin: Springer, 1994.

[9] С. Вейнберг. Гравитация и космология. М.: Мир, 1975.

[10] J. M. Lattimer et al. Phys. Rev. Lett. 1991. V. 66. P. 2701.

[11] G. S. Bisnovatyi-Kogan. Astron. Astrophys. Trans. 1993. V. 3. P. 287.

[12] V. Canuto, H.-Y. Chiu. Phys. Rev. 1968. V. 173. P. 1210, 1220, 1229.

[13] C.-L. Ho, V.R. Khalilov, C. Yang. Mod. Phys. Lett. B. 1996. V. 10. P. 1141

[14] S. Chakrabarty, D. Bandopadhyay, S. Pal. Phys. Rev. Lett. 1997. V. 78. P. 2898.

[15] D. Bandopadhyay, S. Chakrabarty, S. Pal. Phys. Rev. Lett. 1997. V. 79. P. 2176.

[16] A. Broderick, M. Prakash, J. M. Lattimer. Astrophys. J. 2000. V. 537. P. 351.

[17] In Saeng Suh, G. J. Mathews. Cold ideal equation of state for strongly magnetized neutron-star matter. astro-ph/9912301.

[18] M. Chaichian, S. Masood, C. Montonen, A. Pérez Martínez, H. Pérez Rojas. Phys. Rev. Lett. 2000. V. 84. P. 5261; A.P. Martínez, H.P. Rojas, H.J. M. Cuesta. Magnetic collapse of a neutron gas: no magnetar formation. hep-ph/0011399.

[19] В.Р. Халилов. ТМФ. 2002. Т. 130. № 1. С. 87.

[20] W. J. de Haas, P. M. van Alphen. Proc. Amsterdam Acad. 1936. V. 33. Р. 1106; Л. Д. Ландау. Собрание трудов. Т.1. М.: Наука, 1969.

[21] Л. Д. Ландау, Е. М. Лифииц. Статистическая физика. Часть 1. М.: Наука, 1976.

[22] Е. М. Лифииц. Избранные труды. М.: Наука, 1994.

[23] В. Ч. Жуковский, А. С. Виивцев, П. А. Эминов. ЯФ. 1995. Т. 58. С. 1274.

[24] А. С. Виивцев, К. Г. Клименко, Б. В. Магницкий. ЖЭТФ. 1995. Т. 107. С. 307.

[25] А. Д. Балаев и др. ЖЭТФ. 1998. Т. 113. С. 1877.

[26] D. Ebert et al. Phys. Rev. D. 2000. V. 61. P. 025005.

[27] И. М. Тернов, В. Г. Багров, В. Ч. Жуковский. Вестн. Моск. ун-та. Сер. физ., астр. 1966. № 4. C. 21.

[28] J. Schwinger. Phys. Rev. 1948. V. 73. P. 416L; Proc. Nat. Acad. Sci. USA. 1951. V. 37. P. 152,455 .

[29] I. M. Ternov et al. J. Phys. A. 1978. V. 11. P. 739; V.R. Khalilov. Electrons in Strong Electromagnetic Fields. Amsterdam: Gordon and Breach, 1996.

[30] Е. М. Лифuич, Л. П. Питаевский. Статистическая физика. Теория конденсированного состояния. Часть 2. М.: Наука, 1978.

[31] V. R. Khalilov. Phys. Rev. D. 2002. V. 65. P. 056001.

[32] Ч. Киттель. Введение в физику твердого тела. М.: Наука, 1978.

[33] В. Р. Халилов. ТМФ. 2001. Т. 126. С. 427. 